\title{
Left-Ventricular Power-to-Mass Ratio at Peak Exercise Predicts Mortality, Heart Failure, and Cardiovascular Events in Patients with Stable Coronary Artery Disease: Data from the Heart and Soul Study
}

\author{
Ramin Farzaneh-Far ${ }^{a} \quad$ Beeya Na ${ }^{b}$ Mary A. Whooley ${ }^{a} b \quad$ Nelson B. Schiller ${ }^{a} b$ \\ a Department of Medicine, University of California, and ${ }^{b}$ Veterans Affairs Medical Center, San Francisco, Calif., USA
}

\section{Key Words}

Hypertrophy $\cdot$ Coronary $\cdot$ Exercise $\cdot$ Mass $\cdot$ Power

\begin{abstract}
Background: Quantitative stress echocardiography enables calculation of left-ventricular power-to-mass ratio (LVPMR) at peak exercise, a novel measure of cardiac performance per unit mass of myocardial tissue. We hypothesized that LVPMR at peak exercise provides prognostic information beyond established echocardiographic indices such as left-ventricular ejection fraction (LVEF) and left-ventricular mass index (LVMI). Methods: LVPMR (watts/kilogram) at peak exercise was defined as $(k \times$ heart rate $\times$ mean arterial pressure $\times$ stroke volume)/LV mass. We measured LVPMR in 918 adults with stable ambulatory coronary artery disease recruited for the Heart and Soul Study. Hazard ratios (HRs) and 95\% confidence intervals (Cls) were calculated for all-cause mortality, cardiovascular death, nonfatal myocardial infarction, heart failure hospitalization, and combined adverse cardiovascular events. Multivariate adjustments were made for established risk factors including LVEF and LVMI. The prognostic value of LVPMR was also compared with established exercise parameters using receiver-operating characteristic curve analysis. Results: Compared with patients in the highest LVPMR quartile, those in the lowest quartile were at increased risk of all-cause mortality (adjusted HR 1.9; 95\% Cl
\end{abstract}

1.1-3.3), heart failure hospitalization (adjusted HR 2.9;95\% Cl 1.2-6.9), and combined adverse cardiovascular events (adjusted HR 1.9; $95 \% \mathrm{Cl} 1.1-3.4)$. In comparison with the ratepressure product and the Duke treadmill score, LVPMR did not add significant prognostic value ( $p>0.1$ for $c$-statistic comparisons). Conclusions: In patients with stable ambulatory coronary artery disease, LVPMR at peak exercise predicts mortality, heart failure hospitalization, and adverse cardiovascular events. However, LVPMR does not add significant prognostic information beyond established exercise test parameters.

Copyright $\odot 2009$ S. Karger AG, Basel

\section{Introduction}

Hypertrophy of the adult human left ventricle is an adaptive response to increased hemodynamic workload [1]. Large-scale population studies have demonstrated that left-ventricular mass index (LVMI) is an independent predictor of major adverse cardiovascular outcomes including sudden death and the development of congestive heart failure $[2,3]$. However, LVMI is a crude measure of the myocardial response to increased hemodynamic burden, and does not account for potentially favorable alterations in ventricular geometry and myocardial performance [4]. Similarly, although left-ventric-

\section{KARGER}

Fax +4161306 1234 E-Mail karger@karger.ch www.karger.com (c) 2009 S. Karger AG, Basel

0008-6312/09/1143-0226\$26.00/0

Accessible online at:

www.karger.com/crd
Ramin Farzaneh-Far, MD

University of California San Francisco, Box 0124

San Francisco, CA 94143-0124 (USA)

E-Mail rfarzanehfar@medicine.ucsf.edu 
ular ejection fraction (LVEF) has been widely used as a convenient measure of systolic function, almost $50 \%$ of patients admitted with congestive heart failure have a preserved LVEF [5].

An alternative approach to the quantitation of global left-ventricular performance is the calculation of mean cardiac power defined as the product of cardiac output and mean arterial pressure [6]. This calculation incorporates both the flow and pressure-generating functions of the heart in a single quantity. Prior studies have shown that cardiac power is a relatively load-independent measure of left-ventricular performance which is strongly predictive of prognosis in chronic congestive heart failure and cardiogenic shock $[7,8]$. In this study, we defined the left-ventricular power to mass ratio (LVPMR) as the ratio of mean left-ventricular power to left-ventricular mass. By relating cardiac power to mass, the LVPMR reflects global systolic and diastolic performance at the tissue level. We hypothesized that LVPMR provides independent prognostic information regarding adverse cardiovascular events in patients with stable coronary artery disease. We further hypothesized that the prognostic value of LVPMR is independent of LVEF and LVMI in addition to classical risk factors in this patient population.

\section{Methods}

\section{Participants}

The Heart and Soul Study is a prospective cohort study investigating the influence of psychosocial factors on cardiovascular events in outpatients with stable coronary artery disease. The enrollment process for the Heart and Soul Study has been previously described [9]. Eligible participants had at least one of the following: (1) a history of myocardial infarction, (2) angiographic evidence of at least $50 \%$ stenosis by area in at least 1 coronary artery, (3) evidence of exercise-induced ischemia by treadmill electrocardiogram or stress nuclear perfusion imaging, (4) a history of coronary revascularization, or (5) a clinical diagnosis of coronary artery disease as documented by an internist or cardiologist. Individuals were excluded if they deemed themselves unable to walk 1 block, or if they were planning to move out of the local area within 3 years.

Between September 2000 and December 2002, a total of 1,024 participants enrolled. Of these, 80 were unable to perform the exercise treadmill, 26 did not have measurements of LVPMR, and 7 did not provide follow-up information, leaving 911 for outcome analysis. All study participants provided informed consent for stress echocardiographic testing, laboratory testing, and review of medical records. The institutional review board at each of the enrolling centers approved the study.

\section{Baseline Echocardiographic Measurements}

A complete resting 2-dimensional echocardiogram using an Acuson Sequoia ultrasound system (Siemens Medical Solutions, Mountain View, Calif., USA) with a $3.5-\mathrm{MHz}$ transducer and
Doppler ultrasound examination was performed just prior to exercise treadmill testing. Standard 2-dimensional parasternal short-axis and apical 2- and 4-chamber views during inspiration were obtained; these were planimetered using a computerized digitization system to determine end-diastolic (EDV) and endsystolic (ESV) volumes. The LVEF was calculated as (EDV - ESV)/ EDV. Diastolic dysfunction was defined as the presence of at least one of the following: impaired relaxation defined as a ratio of peak mitral early diastolic to atrial contraction velocity (E/A) of $\leq 0.75$ with systolic dominant pulmonary vein flow; pseudonormal defined as $0.75<\mathrm{E} / \mathrm{A}<1.5$ with diastolic dominant pulmonary vein flow; restrictive filling defined as an $\mathrm{E} / \mathrm{A} \geq 1.5$ or greater with diastolic dominant pulmonary vein flow. Left-ventricular mass was calculated using a truncated ellipsoid equation as previously described and validated [10].

\section{Exercise Stress Echocardiogram Measurements}

We performed a symptom-limited graded exercise treadmill test according to a standard Bruce protocol. To achieve maximal heart rate, participants who were unable to continue the standard Bruce protocol were switched to lower settings on the treadmill and encouraged to exercise for as long as possible. Continuous 12lead electrocardiography and monitoring of heart rate and blood pressure were performed throughout. Participants underwent an echocardiogram immediately after exercise. Planimetry with a computerized digitization system was used to obtain EDV and ESV. Stroke volume at peak exercise was calculated as EDV - ESV. The peak rate-pressure product was calculated as the product of the maximal heart rate and blood pressure achieved during exercise. The Duke Treadmill Exercise Score was calculated as described by Mark et al. [11].

\section{Calculation of LVPMR at Peak Exercise}

LVPMR at peak exercise was calculated as: LVPMR (watts/ki$\operatorname{logram})=(\mathrm{k} \times$ heart rate $\times \mathrm{MAP} \times \mathrm{SV}) / \mathrm{LVM}$, where: heart rate $\left(\mathrm{min}^{-1}\right)=$ heart rate at peak exercise; MAP $(\mathrm{mm} \mathrm{Hg})=$ mean arterial pressure at peak exercise; $\mathrm{SV}(\mathrm{ml})=$ stroke volume at peak exercise; $\operatorname{LVM}(\mathrm{g})=$ left-ventricular mass; $\mathrm{k}=$ conversion factor 2.22 $\times 10^{-3}$ (to convert to SI units of watts/kilogram). LVPMR at rest was calculated using the same equation, substituting the resting values of heart rate, mean arterial pressure, and stroke volume.

\section{Other Measurements}

Baseline demographics, self-reported age, sex, ethnicity, medical history, and smoking status were determined by questionnaire. Study participants were instructed to bring their medication bottles to the study appointment where study personnel recorded all current medications. Fasting serum chemistry samples were used to measure HDL cholesterol, LDL cholesterol, and Nterminal pro-B-type natriuretic peptide (NT-proBNP).

\section{Cardiovascular Outcomes}

We conducted annual telephone interviews with participants or their proxies regarding recent emergency room visits, hospitalizations, or death. Medical records, death certificates, and coroner's reports were reviewed by two independent and blinded adjudicators. If the adjudicators agreed on the outcome classification, their classification was binding. If they disagreed, a third blinded adjudicator reviewed the event and determined the outcome classification. 
All-cause mortality was determined by review of death certificates. Death was considered due to cardiovascular causes if the death certificate listed acute myocardial infarction, congestive heart failure, or arrhythmia as the primary cause of death. Sudden death, defined as death occurring unexpectedly within $1 \mathrm{~h}$ of the onset of symptoms, was also considered cardiovascular.

Hospitalization for heart failure was defined as a minimum 1-night hospital stay for a clinical syndrome comprising at least 2 of the following: paroxysmal nocturnal dyspnea, orthopnea, elevated jugular venous pressure, pulmonary rales, third heart sound, and cardiomegaly or pulmonary edema on chest roentgenography. These clinical signs and symptoms must have represented a clear change from the baseline clinical status of the participant and must have been accompanied by either failing cardiac output as determined by peripheral hypoperfusion (in the absence of other causes such as sepsis or dehydration) or peripheral or pulmonary edema requiring intravenous diuretics, inotropes, or vasodilators.

Cardiovascular events were defined as the composite of cardiovascular death, heart failure hospitalization, or nonfatal myocardial infarction. Nonfatal myocardial infarction was defined as hospitalization for acute myocardial infarction as defined by the American Heart Association diagnostic criteria.

\section{Statistical Analyses}

We included 918 participants with completed outcome adjudications in the analyses. Because age-, sex-, and race-specific normal ranges for LVPMR have not been established, we categorized LVPMR into quartile groups. Differences in baseline characteristics were compared with the use of ANOVA for continuous variables and the $\chi^{2}$ test for dichotomous variables. We used multivariate logistic regression analysis to calculate hazard ratios (HRs) for quartiles of LVPMR as the primary predictor variable and outcomes. We report HRs with 95\% confidence intervals (95\% CIs). Predefined end points were all-cause mortality, cardiovascular death, heart failure hospitalization, nonfatal heart failure, and the combined end point (composite of cardiovascular death, heart failure hospitalization, and nonfatal myocardial infarction). To determine the independent association between LVPMR and outcomes, we adjusted for known clinical risk factors (age, sex, smoking status, hypertension, diabetes, body mass index, body surface area, history of myocardial infarction, history of chronic heart failure, HDL cholesterol), echocardiographic risk factors (LVEF, LVMI), and $\beta$-blocker use. We used receiver-operating characteristic curve analysis, and calculation of c-statistic, to compare the predictive value of LVPMR at peak exercise with other established prognostic markers. Statistical analysis was performed using SAS software version 9.1 (SAS Institute Inc., Cary, N.C., USA).

\section{Results}

The LVPMR at peak exercise in the study population ranged from 0.95 to $38 \mathrm{~W} / \mathrm{kg}$. Baseline characteristics of the 918 study participants by LVPMR quartile are shown in table 1. Compared with participants in the highest quartile of peak LVPMR ( $>12 \mathrm{~W} / \mathrm{kg})$, those in the lowest quartile $(<6.7 \mathrm{~W} / \mathrm{kg})$ were more likely to be older, to have co-morbid medical conditions (obesity, hypertension, prior chronic heart failure, diabetes), to have lower exercise capacity, and to be taking $\beta$-blockers. They had lower levels of HDL cholesterol, higher levels of B-type natriuretic peptide, lower LVEF, and higher LVMI. There was no significant difference in gender, ethnicity, or diastolic dysfunction across quartiles of LVPMR.

During a mean follow-up of 4.2 years, there were 160 deaths, 45 cardiovascular deaths, 96 hospitalizations for heart failure, 79 nonfatal myocardial infarctions, and 164 cardiovascular events (cardiovascular death, heart failure hospitalization or nonfatal myocardial infarction). The number of participants with outcome events separated by LVPMR quartile, and the corresponding unadjusted HRs are shown in table 2. Outcome by quartiles of LVPMR is shown graphically in figure 1.

LVPMR was independently predictive of all-cause mortality, heart failure hospitalization, and cardiovascular events even after adjusting for known clinical and echocardiographic prognostic markers. Multivariate regression analysis adjusted for age, sex, smoking status, hypertension, diabetes, BMI, BSA, history of myocardial infarction, history of CHF, HDL cholesterol, LVEF, LV mass index, and beta-blocker use is shown in table 3 . When all-cause mortality, cardiovascular death, heart failure hospitalization, nonfatal myocardial infarction, and cardiovascular events were evaluated as individual outcome measures, the adjusted HRs for participants in quartile I compared to quartile IV were 1.9 (95\% CI 1.11.3) for all-cause mortality, 1.7 (95\% CI 0.6-4.8) for cardiovascular death, 2.9 (95\% CI 1.2-6.9) for heart failure hospitalization, 1.5 (95\% CI 0.7-3.1) for nonfatal myocardial infarction, and 1.9 (95\% CI 1.1-3.4) for cardiovascular events. For each SD decrease in LVPMR the adjusted HRs for outcomes were as follows: all-cause mortality $\mathrm{HR}=1.3(95 \% \mathrm{CI} 1.0-1.6)$, cardiovascular death $\mathrm{HR}=1.3$ (95\% CI 0.9-2.0), heart failure hospitalization $\mathrm{HR}=1.5$ (95\% CI 1.1-2.0), nonfatal myocardial infarction $\mathrm{HR}=1.3$ (95\% CI 1.0-1.8), cardiovascular events HR $=1.3(95 \%$ $\mathrm{CI}=1.1-1.7)$. The adjusted HRs for independent prediction of cardiovascular death and nonfatal myocardial infarction did not reach statistical significance. Survival free of cardiovascular events by LVPMR quartile is shown in figure 2.

The receiver-operating characteristic curve for the prediction of heart failure hospitalization by LVPMR and alternative exercise parameters is shown in figure 3 . The c-statistic for each outcome by predictor is summarized in table 4. 
Table 1. Baseline characteristics of study participants by quartile of LVPMR

\begin{tabular}{|c|c|c|c|c|c|}
\hline \multirow[t]{2}{*}{ Variable } & \multicolumn{4}{|c|}{ LVPMR quartiles, W/kg } & \multirow[t]{2}{*}{$\mathrm{p}$ value } \\
\hline & $\begin{array}{l}\text { quartile } 1 \\
(1.0-6.7) \\
(\mathrm{n}=229)\end{array}$ & $\begin{array}{l}\text { quartile } 2 \\
(6.7-9.2) \\
(n=230)\end{array}$ & $\begin{array}{l}\text { quartile } 3 \\
(9.2-12.0) \\
(\mathrm{n}=229)\end{array}$ & $\begin{array}{l}\text { quartile } 4 \\
(12.0-38.0) \\
(n=230)\end{array}$ & \\
\hline Age, years & $69 \pm 11$ & $67 \pm 10$ & $66 \pm 11$ & $65 \pm 11$ & $<0.0001$ \\
\hline Male sex & $197(86 \%)$ & $182(79 \%)$ & $193(84 \%)$ & $190(83 \%)$ & 0.2 \\
\hline Body mass index & $29 \pm 5$ & $29 \pm 5$ & $28 \pm 5$ & $27 \pm 5$ & 0.0006 \\
\hline Body surface area, $\mathrm{m}^{2}$ & $2.0 \pm 0.2$ & $2.0 \pm 0.2$ & $1.9 \pm 0.2$ & $1.9 \pm 0.2$ & 0.04 \\
\hline Ethnicity & & & & & 0.1 \\
\hline White & $142(62 \%)$ & $129(56 \%)$ & $132(58 \%)$ & $154(67 \%)$ & \\
\hline Black & $33(14 \%)$ & $47(21 \%)$ & $33(14 \%)$ & $30(13 \%)$ & \\
\hline Asian & $27(12 \%)$ & $22(10 \%)$ & $31(14 \%)$ & $27(12 \%)$ & \\
\hline Other & $27(12 \%)$ & $31(14 \%)$ & $33(14 \%)$ & $19(8 \%)$ & \\
\hline Hypertension & $181(79 \%)$ & $169(73 \%)$ & $161(71 \%)$ & $130(57 \%)$ & $<0.0001$ \\
\hline Myocardial infarction & $134(59 \%)$ & $120(52 \%)$ & $125(55 \%)$ & $106(46 \%)$ & 0.05 \\
\hline Chronic heart failure & $66(29 \%)$ & $35(15 \%)$ & $29(13 \%)$ & $20(9 \%)$ & $<0.0001$ \\
\hline Stroke & $39(17 \%)$ & $32(14 \%)$ & $31(14 \%)$ & $22(10 \%)$ & 0.1 \\
\hline Diabetes & $70(31 \%)$ & $70(30 \%)$ & $50(22 \%)$ & $37(16 \%)$ & 0.0004 \\
\hline Revascularization & $150(66 \%)$ & $127(55 \%)$ & $141(62 \%)$ & $136(59 \%)$ & 0.1 \\
\hline Current smoking & $51(22 \%)$ & $48(21 \%)$ & $49(21 \%)$ & $33(14 \%)$ & 0.1 \\
\hline LVEF & $59 \pm 11$ & $62 \pm 10$ & $63 \pm 8$ & $64 \pm 8$ & $<0.0001$ \\
\hline Diastolic dysfunction & & & & & 0.2 \\
\hline Impaired relaxation & $121(80 \%)$ & $124(86 \%)$ & $106(80 \%)$ & $110(85 \%)$ & \\
\hline Pseudonormal & $28(18 \%)$ & $16(11 \%)$ & $18(14 \%)$ & $13(10 \%)$ & \\
\hline Restrictive & $4(3 \%)$ & $4(3 \%)$ & $9(7 \%)$ & $6(5 \%)$ & \\
\hline LV mass, $\mathrm{g}$ & $225 \pm 58$ & $198 \pm 57$ & $177 \pm 41$ & $156 \pm 35$ & $<0.0001$ \\
\hline LVMI, $\mathrm{g} / \mathrm{m}^{2}$ & $114 \pm 27$ & $100 \pm 25$ & $92 \pm 19$ & $81 \pm 17$ & $<0.0001$ \\
\hline LVESV, ml & $47 \pm 33$ & $39 \pm 24$ & $39 \pm 22$ & $37 \pm 20$ & $<0.0001$ \\
\hline LVEDV, $\mathrm{ml}$ & $108 \pm 44$ & $98 \pm 34$ & $102 \pm 35$ & $99 \pm 31$ & 0.01 \\
\hline Stroke volume, $\mathrm{ml}$ & $60 \pm 18$ & $59 \pm 16$ & $63 \pm 17$ & $62 \pm 17$ & 0.2 \\
\hline Heart rate, $\min ^{-1}$ & $65 \pm 11$ & $68 \pm 12$ & $68 \pm 12$ & $68 \pm 12$ & 0.01 \\
\hline Mean arterial pressure, $\mathrm{mm} \mathrm{Hg}$ & $92 \pm 12$ & $94 \pm 12$ & $94 \pm 11$ & $93 \pm 12$ & 0.4 \\
\hline Exercise capacity, METS & $5.4 \pm 2.5$ & $6.8 \pm 3.0$ & $7.8 \pm 2.8$ & $9.5 \pm 3.5$ & $<0.0001$ \\
\hline Current $\beta$-blocker use & $166(72 \%)$ & $142(62 \%)$ & $127(55 \%)$ & $100(43 \%)$ & $<0.0001$ \\
\hline log NTproBNP & $5.8 \pm 1.4$ & $5.2 \pm 1.2$ & $5.0 \pm 1.2$ & $4.7 \pm 1.1$ & $<0.0001$ \\
\hline LDL cholesterol, mg/dl & $102 \pm 34$ & $103 \pm 29$ & $104 \pm 35$ & $106 \pm 35$ & 0.6 \\
\hline HDL cholesterol, mg/dl & $43 \pm 12$ & $46 \pm 13$ & $46 \pm 15$ & $49 \pm 15$ & $<0.0001$ \\
\hline
\end{tabular}

\section{Discussion}

We demonstrate in this study that LVPMR at peak exercise, a simple and novel measure of myocardial performance, predicts mortality, heart failure hospitalization, and cardiovascular outcomes independently of traditional cardiac risk factors and other echocardiographic indices in patients with stable ambulatory coronary artery disease. However, we did not observe any incremental prognostic value beyond regular exercise test parameters such as the rate-pressure product and the Duke treadmill score.

Left-Ventricular Power-to-Mass Ratio

\section{Limitations of LVEF and LVMI in Risk Stratification}

There is a need for improvement in objective risk stratification in patients with coronary artery disease to determine the optimal indications for targeted therapies such as implantable defibrillator therapy, biventricular pacing, and aggressive afterload reduction [12, 13]. Although both LVEF and LVMI have been shown to be predictive of adverse cardiovascular events, these measures have significant limitations. LVMI is a crude measure of the myocardial response to increased hemodynamic burden and does not account for potentially favorable alterations in ventricular geometry and myocardial perfor- 
Fig. 1. Outcome by quartiles of LVPMR.

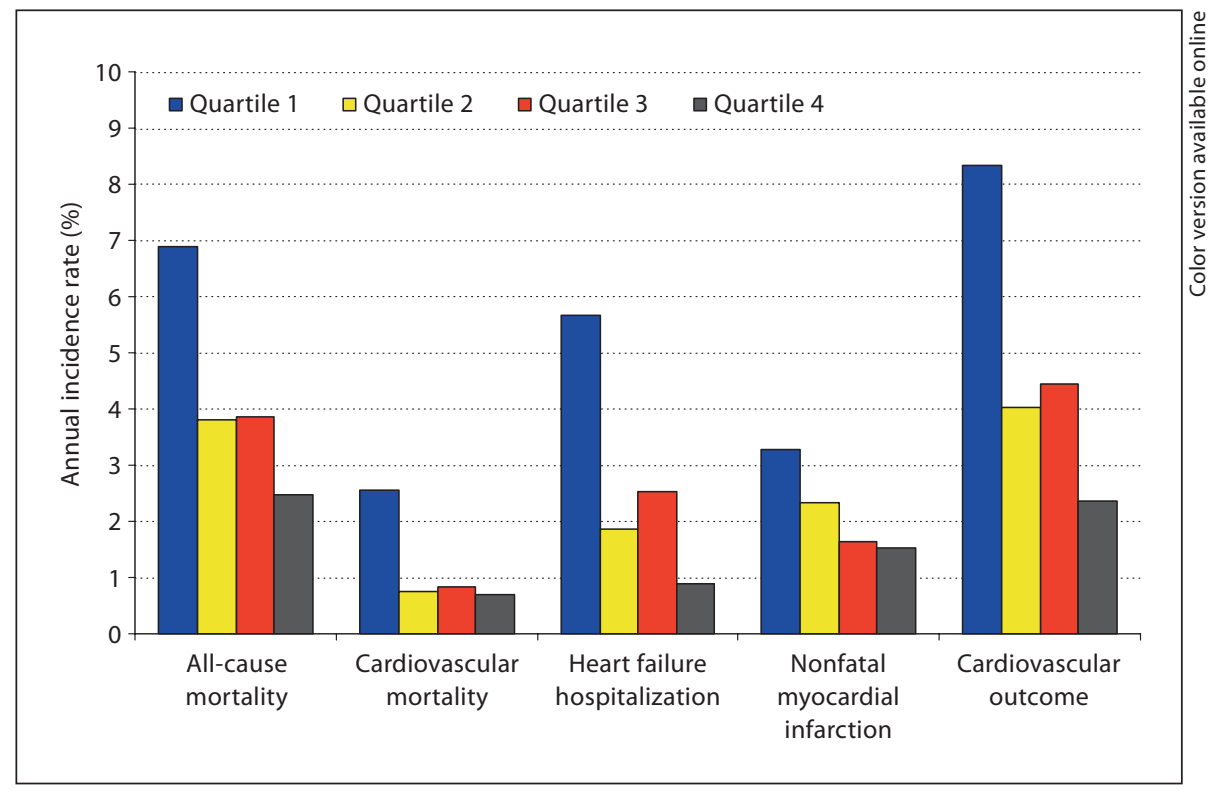

Table 2. Adverse cardiovascular outcomes by quartile of LVPMR

\begin{tabular}{|c|c|c|c|c|c|c|}
\hline & \multicolumn{4}{|c|}{ LVPMR quartiles, W/kg } & \multirow[t]{2}{*}{$\mathrm{HR}^{1}, 95 \% \mathrm{CI}$} & \multirow{2}{*}{$\begin{array}{l}\mathrm{p} \\
\text { value }\end{array}$} \\
\hline & $\begin{array}{l}\text { quartile } 1 \\
(0.95-6.68)\end{array}$ & $\begin{array}{l}\text { quartile } 2 \\
(6.69-9.20)\end{array}$ & $\begin{array}{l}\text { quartile } 3 \\
(9.22-12.12)\end{array}$ & $\begin{array}{l}\text { quartile } 4 \\
(12.15-38.03)\end{array}$ & & \\
\hline All-cause mortality & $62(27 \%)$ & $36(16 \%)$ & $37(16 \%)$ & $25(1$ & $3.0,1.9-4.8$ & $<0.001$ \\
\hline Cardiovascular death & $23(10 \%)$ & $7(3 \%)$ & $8(4 \%)$ & $7(3 \%)$ & $3.8,1.6-8.8$ & 0.002 \\
\hline Heart failure hospitalization & $47(21 \%)$ & $17(7 \%)$ & $23(10 \%)$ & $9(4 \%)$ & $6.2,3.0-12.6$ & $<0.001$ \\
\hline Nonfatal myocardial infarction & $28(12 \%)$ & $21(9 \%)$ & $15(7 \%)$ & $15(7 \%)$ & $2.2,1.2-4.0$ & 0.02 \\
\hline Cardiovascular events & $67(29 \%)$ & $35(15 \%)$ & $39(17 \%)$ & $23(10 \%)$ & $3.5,2.2-5.6$ & $<0.001$ \\
\hline
\end{tabular}

${ }^{1}$ Quartile 1 versus quartile 4.

mance. LVEF is a widely used and convenient measure of resting systolic function, and is currently the dominant echocardiographic parameter of long-term prognosis in patients with coronary heart disease and heart failure. However, LVEF is a relatively poor predictor of sudden cardiac death, and approximately two-thirds of patients who undergo implantable defibrillator placement based on LVEF never have an appropriate shock [14]. Furthermore, LVEF is of little value in the diagnosis and prognostication of patients admitted with diastolic heart failure, a group that accounts for almost one-half of heart failure hospitalizations. In this group of patients, chronotropic incompetence, impaired vasodilatation with exercise and increased left-atrial volume have recently been demonstrated to be predictive of symptomatic heart failure $[15,16]$.

\section{Concept: Power-to-Mass Ratios and 'Cardiac \\ Horsepower'}

Power-to-mass ratios are widely applied in engineering, aeronautics, and the automobile industry to compare the performance of vehicles, aircraft, and engines. In the human heart, useful power output is generated by cardiac muscle which undergoes compensatory hypertrophy in response to increased hemodynamic workload. In terms of myocardial energetics, a heart which maintains normal pump function at the expense of hypertrophy is not as efficient at the tissue level as one which maintains 
Fig. 2. Survival free of cardiovascular events (cardiovascular death, heart failure hospitalization, or nonfatal myocardial infarction) by LVPMR quartile.

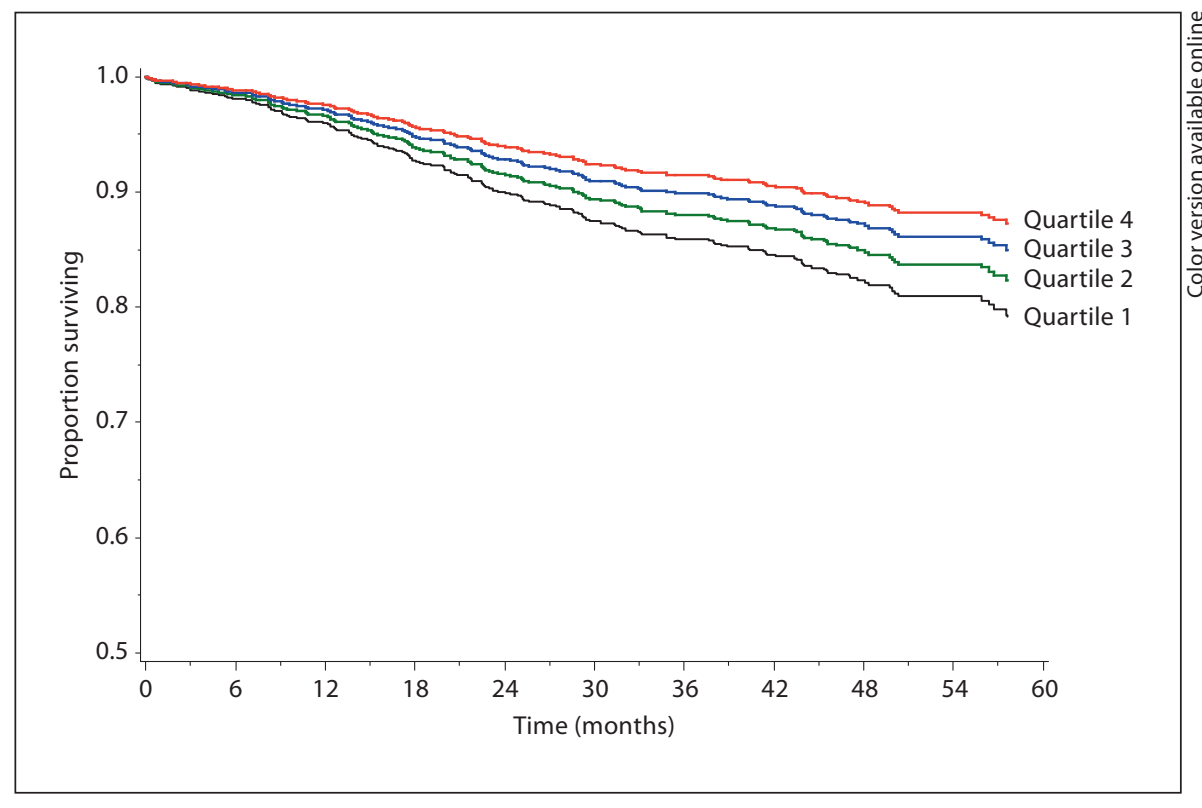

Fig. 3. ROC curves for the prediction of heart failure hospitalization by LVPMR at peak exercise, LVPMR at rest, Duke treadmill score, and the rate-pressure product.

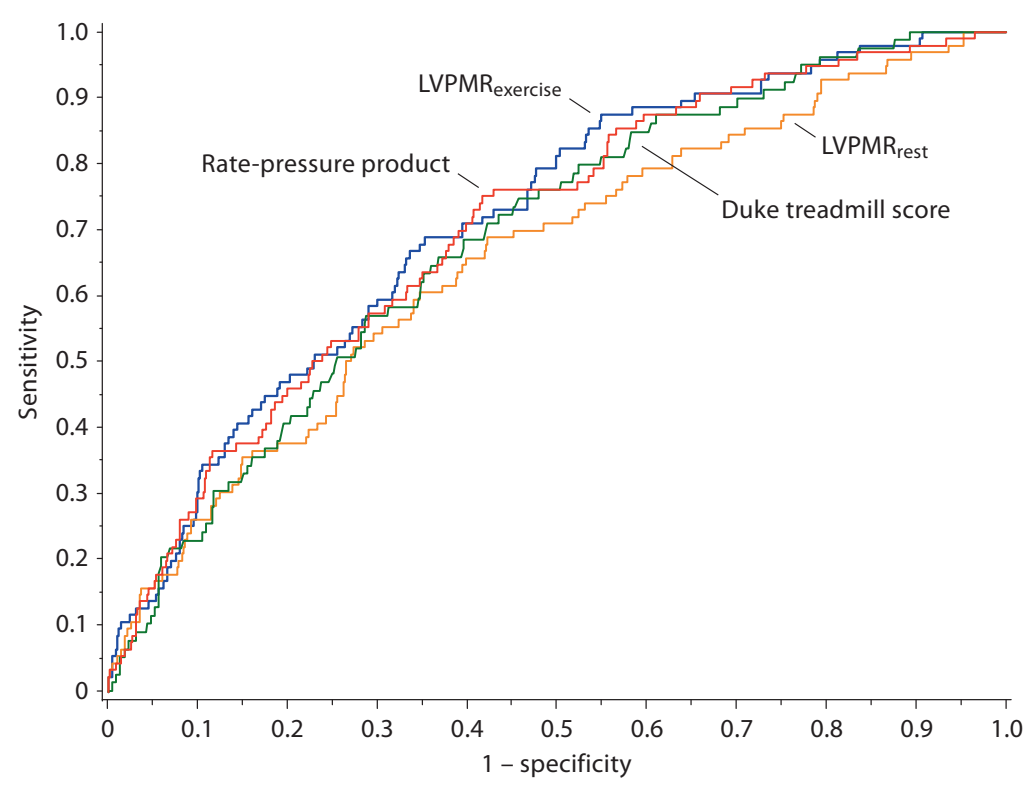

normal pump function without hypertrophy [17]. We applied this intuitive principle to the pump function of the human heart by defining LVPMR as the maximal cardiac power output divided by left-ventricular mass, thus providing a measure of composite systolic and diastolic performance which is both relatively load-independent, and accounts for pathologic left-ventricular hypertrophy. Physiologic left-ventricular hypertrophy such as that which occurs in elite athletes would not be expected to reduce LVPMR as maximal power output would be expected to increase exponentially compared to mass. LVPMR can thus be considered conceptually equivalent to 'cardiac horsepower'.

\section{Advantages of LVPMR}

We show that LVPMR is predictive of all-cause mortality, heart failure hospitalization, and cardiovascular events in patients with ambulatory coronary artery disease even 
Table 3. Association of LVPMR with adverse cardiovascular events after adjustment for available clinical and echocardiographic risk factors (including LVEF and LVMI)

\begin{tabular}{lllll}
\hline & $\mathrm{HR}^{1}, 95 \% \mathrm{CI}$ & $\mathrm{p}$ & $\mathrm{HR}^{2}, 95 \% \mathrm{CI}$ & $\mathrm{p}$ \\
\hline $\begin{array}{l}\text { All-cause mortality } \\
\text { Cardiovascular death }\end{array}$ & $1.9,1.1-3.3$ & 0.02 & $1.3,1.0-1.6$ & 0.02 \\
$\begin{array}{l}\text { Heart failure hospital- } \\
\quad \text { ization }\end{array}$ & $1.7,0.6-4.8$ & 0.3 & $1.3,0.9-2.0$ & 0.8 \\
$\begin{array}{l}\text { Nonfatal myocardial } \\
\quad \text { infarction }\end{array}$ & $1.9,1.2-6.9$ & 0.01 & $1.5,1.1-2.0$ & 0.02 \\
$\begin{array}{l}\text { Cardiovascular events } \\
\text { ardioval }\end{array}$ & $1.9,1.1-3.4$ & 0.02 & $1.3,1.1-1.7$ & 0.01 \\
\hline
\end{tabular}

${ }^{1}$ Quartile 1 vs. $4 ;{ }^{2}$ per SD decrease.

Table 4. C-statistic for the prediction of adverse outcomes by potential prognostic parameters

\begin{tabular}{llll}
\hline & Death & Heart failure & $\begin{array}{l}\text { Cardiovas- } \\
\text { cular events }\end{array}$ \\
\hline LVPMR $_{\text {exercise }}$ & 0.69 & 0.71 & 0.69 \\
Rate-pressure product & 0.70 & 0.70 & 0.69 \\
Duke Treadmill Score $_{\text {LVPMR }_{\text {rest }}}$ & 0.69 & 0.69 & 0.69 \\
\hline
\end{tabular}

${ }^{*} \mathrm{p}<0.05$ for comparison with LVPMR exercise.

after adjustment for traditional risk factors and echocardiographic indices including LVEF and LVMI. LVPMR therefore provides incremental and independent prognostic information regarding adverse cardiovascular events in patients with stable coronary artery disease over and above LVEF and LVMI. We suggest that LVPMR may enable refinement of the indications for targeted therapies in stable coronary artery disease and heart failure, whether systolic or diastolic. Another advantage of LVPMR is its relative load-independence as a measure of systolic and diastolic performance, which renders it potentially more informative than LVEF as a therapeutic end point in studies of novel heart failure therapies [18]. LVPMR can be calculated easily from parameters obtained during quantitative stress echocardiography (stroke volume, heart rate, mean arterial pressure and left-ventricular mass). Further clinical studies are required to determine the value of LVPMR in risk stratification and therapy selection, and to determine the effect of medical therapies on LVPMR.
Although previous studies have shown that cardiac power output is strongly predictive of prognosis in chronic congestive heart failure and cardiogenic shock, this study is the first to relate cardiac power to left-ventricular mass, and further to show that LVPMR predicts all-cause mortality, heart failure hospitalizations, and cardiovascular events in patients with stable ambulatory coronary artery disease.

As a global measure of systolic and diastolic performance corrected for left-ventricular mass, LVPMR has many other potential clinical applications such as the evaluation of patients with hypertrophic, restrictive, and dilated cardiomyopathies, and the study of physiologic hypertrophy in athletes.

\section{Comparison of LVMPR with Established Prognostic Parameters}

Our results indicate that LVPMR at peak exercise offers no incremental prognostic value in the prediction of mortality, heart failure, and adverse cardiovascular events compared with the Duke Treadmill Score and the rate-pressure product. However, changes in the c-statistic, which achieve statistical significance, are notoriously difficult to observe, even when the predictor of interest improves reclassification indices [19]. Nevertheless, for the time being, LVPMR at peak exercise should be considered of little independent clinical significance.

\section{Study Limitations}

Several limitations of the present study must be considered. First, the calculation of LVPMR was based on echocardiographic and hemodynamic parameters achieved during a symptom-limited exercise stress test, and required participants to exercise to capacity. Not all participants stopped exercise due to cardiovascular symptoms of chest pain, dyspnea, or syncope. A substantial number of patients stopping exercise due to noncardiac reasons would be expected to reduce the predictive ability of LVPMR for cardiovascular outcomes. Second, our calculations of stroke volume and left-ventricular mass were based on geometric assumptions which, although validated and accurate, are less precise than those obtained by cardiac magnetic resonance imaging, the current gold standard for volumetric and left-ventricular mass analysis [20,21]. In particular, volumetric analysis by echocardiography underestimates stroke volume relative to magnetic resonance imaging [22]. An alternative approach would be to use the aortic Doppler velocity- 
time integral at peak exercise as a surrogate for stroke volume, a technique which also has significant limitations [23]. Third, the adjusted HRs for independent prediction of cardiovascular death and nonfatal myocardial infarction by LVPMR did not reach statistical significance. The relatively small number of adjudicated cardiovascular deaths in this study (45 patients) could explain the lack of statistical significance for this outcome. Regarding nonfatal myocardial infarction, we did not incorporate inducible wall motion abnormalities with exercise into our analysis which would provide additional prognostic information [24, 25]. Fourth, our study was restricted to patients with ambulatory stable coronary artery disease. The test characteristics of LVPMR in this patient population cannot necessarily be extended to patients with nonischemic cardiomyopathy, hypertrophic cardiomyopathy, or valvular heart disease.

\section{Conclusions}

LVPMR is a novel measure of global myocardial performance, which predicts mortality, heart failure hospitalization, and cardiovascular events in patients with sta- ble coronary artery disease. It does not add to the prognostic utility of established exercise parameters such as the Duke Treadmill Score and the rate-pressure product. Further studies are needed to determine whether there is any role for LVPMR in risk stratification, clinical decision-making, and therapy selection in patients with coronary artery disease. As a relatively load-independent global measure of combined systolic and diastolic performance, LVPMR may have further specific applications in the study of dilated, restrictive, and hypertrophic cardiomyopathies.

\section{Acknowledgements}

The Heart and Soul study was supported by the Department of Veterans Affairs (Epidemiology Merit Review Program), the Robert Wood Johnson Foundation (Generalist Physician Faculty Scholars Program), the American Federation for Aging Research (Paul Beeson Faculty Scholars in Aging Research Program), and the Nancy Kirwan Heart Research Fund. R.F.-F. is supported by an American Heart Association Fellow-to-Faculty Transition Award.

\section{References}

-1 De Simone G, Devereux RB, Kimball TR, Mureddu GF, Roman MJ, Contaldo F, et al: Interaction between body size and cardiac workload: influence on left ventricular mass during body growth and adulthood. Hypertension 1998;31:1077-1082.

-2 Elhendy A, Modesto KM, Mahoney DW, Khanderia BK, Seward JB, Pellikka PA: Prediction of mortality in patients with left ventricular hypertrophy by clinical, exercise stress, and echocardiographic data. J Am Coll Cardiol 2003;41:129-135.

-3 Hawkins NM, Wang D, McMurray JJV, Pfeffer MA, Swedberg K, Granger CB, et al: Prevalence and prognostic implications of electrocardiographic left ventricular hypertrophy in heart failure: evidence from the CHARM programme. Heart 2007;93:5964.

4 De Castro S, Caselli S, Maron M, Peliccia A, Cavarretta E, Maddukuri P, et al: Left ventricular remodelling index (LVRI) in various patho-physiological conditions: a real-time three-dimensional echocardiographic study. Heart 2007;93:205-209.
5 Owan TE, Hodge TE, Herges RM, Jacobsen SJ, Roger VL, Redfield MM: Trends in prevalence and outcome of heart failure with preserved ejection fraction. N Engl J Med 2006; 355:251-259.

6 Armstrong GP, Carlier SG, Fukamachi K, Thomas JD, Marwick TH: Estimation of cardiac reserve by peak power: validation and initial application of a simplified index. Heart 1999;82:357-364.

7 Williams SG, Cooke GA, Wright DJ, Parsons WJ, Riley RL, Marshall P, et al: Peak exercise cardiac power output: a direct indicator of cardiac function strongly predictive of prognosis in chronic heart failure. Eur Heart J 2001;22:1496-1503.

-8 Fincke R, Hochman JS, Lowe AM, Menon V, Slater J, Webb JG, et al: Cardiac power is the strongest hemodynamic correlate of mortality in cardiogenic shock: a report from the SHOCK Trial Registry. J Am Coll Cardiol 2004;44:340-348.

$\checkmark 9$ Ruo B, Rumsfeld JS, Hlatky MA, Liu H, Browner WS, Whooley MA: Depressive symptoms and health-related quality of life: the Heart and Soul Study. JAMA 2003;290: 215-221.
10 Schiller NB, Skioldebrand CG, Schiller EJ, Mavroudis CC, Silverman NH, Rahimtoola $\mathrm{SH}$, et al: Canine left ventricular mass estimation by two-dimensional echocardiography. Circulation 1983;68:210-216.

11 Mark DB, Shaw L, Harrell FE Jr, Hlatky MA, Lee KL, Bengston JR, et al: Prognostic value of a treadmill exercise score in outpatients with suspected coronary artery disease. N Engl J Med 1991;325:887-888.

12 Buxton AE: Identifying the high risk patient with coronary artery disease - is ejection fraction all you need? J Cardiovasc Electrophysiol 2005(suppl 1):25-27.

$>13$ Shaw LJ, Hachamovitch R, Heller GV, Marwick TH, Travin MI, Iskandrian, AE, et al: Noninvasive strategies for the estimation of cardiac risk in stable chest pain patients. The Economics of Noninvasive Diagnosis (END) Study Group. Am J Cardiol 2000;86:1-7.

14 Desai AS, Stevenson LW: Selecting patients for discussion of the ICD as primary prevention for sudden death in heart failure. J Card Fail 2006;12:407-412. 
-15 Borlaug B, Melenovsky V, Russell SD, Kessler K, Pacak K, Becker LC, et al: Impaired chronotropic and vasodilator reserve limits exercise capacity in patients with heart failure and a preserved ejection fraction. Circulation 2006;114:2138-2147.

16 Melenovsky B, Borlaug BA, Rosen B, Hay I, Ferucci L, Morell CH, et al: Cardiovascular features of heart failure with preserved ejection fraction versus nonfailing hypertensive left ventricular hypertrophy im the urban Baltimore community: the role of atrial remodeling/dysfunction. J Am Coll Cardiol 2007:49:198-207.

-17 Knaapen P, Germans T, Knuuti J, Paulus WJ, Dijkmans PA, Allaart CP, et al: Myocardial energetics and efficiency: current status of the noninvasive approach. Circulation 2007; 115:918-927.
18 Marwick TH: Techniques for comprehensive two-dimensional echocardiographic assessment of left ventricular systolic function. Heart 2003;83(suppl 3):2-8

19 Zou KH, O’Malley AJ, Mauri L: Receiver-operating characteristic analysis for evaluating diagnostic tests and predictive models. Circulation 2007;115:654-657

20 Pattnyama PM, De Roos A, Van der Wall EE, Van Voorthuisen AE: Evaluation of cardiac function with magnetic resonance imaging. Am Heart J 1994;128:595-607.

21 Chuang Ml, Hibberd MG, Salton CJ, Beaudin RA, Riley MF, Parker RA, et al: Importance of imaging method over imaging modality in noninvasive determination of left ventricular volumes and ejection fraction: assessment by two- and three-dimensional echocardiography and magnetic resonance imaging. J Am Coll Cardiol 2000;35:477484.

22 Malm S, Frigstad S, Sagberg E, Larsson H, Skjaerpe T: Accurate and reproducible measurement of left ventricular volume and ejection fraction by contrast echocardiography: a comparison with magnetic resonance imaging. J Am Coll Cardiol 2004;44:10301035.
23 Haugen BO, Berg S, Brecke KM, Samstad SO, Skjaerpe T, Slordahl SA, et al: Blood flow velocity profiles in the aortic annulus: a 3-dimensional freehand color flow Doppler imaging study. J Am Soc Echocardiogr 2002;15: 328-333.

-24 Metz LD, Beattie M, Hom R, Redberg RG, Grady D, Fleischmann KE: The prognostic value of normal exercise myocardial perfusion imaging and exercise echocardiography: a meta-analysis. J Am Coll Cardiol 2007;49:227-237.

25 Peteiro J, Monserrat L, Pineiro M, Calvino R, Vazquez JM, Marinas J, et al: Comparison of exercise echocardiography and the Duke treadmill score for risk stratification in patients with known or suspected coronary artery disease and normal resting electrocardiogram. Am Heart J 2006;151:1324.e11324.e10. 